\title{
Entanglement switching via the Kondo effect in triple quantum dots
}

\author{
S.B. Tooski ${ }^{1,2}$, Bogdan R. Bułka ${ }^{1, a}$, Rok Žitko ${ }^{2}$, and Anton Ramšak ${ }^{2}$ \\ 1 Institute of Molecular Physics, Polish Academy of Sciences, ul. M. Smoluchowskiego 17, 60-179 Poznań, Poland \\ 2 Faculty of Mathematics and Physics, University of Ljubljana and Jožef Stefan Institute, 1000 Ljubljana, Slovenia
}

Received 21 November 2013 / Received in final form 14 April 2014

Published online 25 June 2014

(C) The Author(s) 2014. This article is published with open access at Springerlink.com

\begin{abstract}
We consider a triple quantum dot system in a triangular geometry with one of the dots connected to metallic leads. Using Wilson's numerical renormalization group method, we investigate quantum entanglement and its relation to the thermodynamic and transport properties in the regime where each of the dots is singly occupied on average, but with non-negligible charge fluctuations. It is shown that even in the regime of significant charge fluctuations the formation of the Kondo singlets induces switching between separable and perfectly entangled states. The quantum phase transition between unentangled and entangled states is analyzed quantitatively and the corresponding phase diagram is explained by exactly solvable spin model. In the framework of an effective model we also explain smearing of the entanglement transition for cases when the symmetry of the triple quantum dot system is relaxed.
\end{abstract}

\section{Introduction}

Creation of entangled states is essential for quantum computation and communication where most qubit operations cannot be performed through the manipulation of separable states [1-4]. Two electron spin qubits, each localized in one of two adjacent semiconductor quantum dots (QDs), can be coupled via the Heisenberg exchange interaction due to virtual electron tunneling between the dots $[5,6]$. Following the analysis of the use of entangled electron spin pairs in solid-state structures [7], an intense activity was aimed toward understanding the physical mechanisms that produce spin-entangled electrons in mesoscopic conductors.

The coherent manipulation of a single electron spin in a QD and the controlled correlation of two spins located in isolated dots have already been demonstrated experimentally [8]. Three coherently coupled quantum dots with three spins attracted interest in the hope to construct a qubit using purely electrically gated manipulations $[9,10]$. Most recently, for a triple quantum dot (TQD) structure it was shown that bipolar spin blockade can become a relevant quantum coherent mechanism [11].

The interaction of qubit pairs with the environment is in general a complicated many-body process and its understanding is essential for experimental and theoretical solidstate qubits [12-16]. Such a system is often represented by a two-level system, or spin-1/2, interacting with an otherwise homogeneous, and often one-dimensional medium with gapless excitations. Some versions of this model

\footnotetext{
a e-mail: bulka@ifmpan.poznan.pl
}

are equivalent to the Kondo model, motivating studies of ground state entanglement of an impurity spin with the conduction electrons and the role of the Kondo effect. Entanglement can also be generated by scattering of strongly interacting electrons [17] or in traps of surface acoustic waves [18]. In general the entanglement can be easily expressed exactly in terms of the impurity magnetization [12-16,19-25]. It was found that the Kondo effect plays a significant role in spin qubit double-quantum dot (DQD) [19-21].

The description of electrons by the spin degrees of freedom alone is a simplification valid when the electrons are localized and the charge fluctuations are negligible. In general, in any realistic solid-state device, spin entanglement is closely connected to the orbital degrees of freedom of the carriers which can be traced out if not measured [26,27].

The production and manipulation of spin qubit pair entanglement has been in TQDs studied for various QD configurations and mechanisms [28-31]. The transport properties of TQDs in various configurations have also been investigated [32-41]. In a serial TQD, different Kondo regimes can be sampled by measuring the conductance. The transition from local-moment to molecularorbital regime can be observed in the evolution of correlation functions [32]. A sizable splitting of the Kondo resonance and a two-stage screening of the magnetic moment was found in a TQD system containing a central dot attached to the leads [35]. The inter-dot hopping introduces the Kondo-assisted transport and may induce a quantum phase transition $[37,38]$. The possibility of a robust underscreened Kondo effect in TQD has also been recently discussed [41]. 


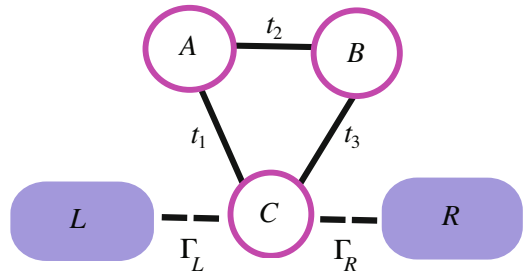

Fig. 1. Triple quantum dot system attached to the leads.

In this work we concentrate on the influence of thermodynamic and transport properties on the entanglement of electrons confined in three adjacent quantum dots forming a triangle and with one of the dots attached to two metallic leads, Figure 1. We show that by breaking the triangular symmetry of the system one can change superexchange interactions and the entanglement of electrons, but on the other hand, the coupling to the leads induces the Kondo cloud which can switch the inter-dot spin-spin correlations due to the interplay between various Kondo-like ground states. We report here how the Kondo interaction can lead to either a collapse of entanglement or can generate fully entangled states between the dots. The results differ from those most typical in the DQD configurations in which the Kondo interaction reduces the entanglement between the DQD qubits [19-21].

The outline of the paper is as follows. Section 2 presents the model and the numerical renormalizationgroup (NRG) method. Section 3 introduces a measure of entanglement. In Sections 4 and 5 the main results of the paper are presented, with numerical analysis summarized in a phase diagram on an entanglement switching in the considered TQD system.

\section{Model and method}

We consider a solid-state qubit system built from three quantum dots attached to non-interacting leads, as is shown in Figure 1. We model the system by the threeimpurity Anderson Hamiltonian and we are interested in the regime, where each of the dots is singly occupied.

The Hamiltonian consists of three parts: $H=H_{d}+$ $H_{\text {lead }}+H_{t}$. The isolated dots are described by

$H_{d}=\sum_{i} \epsilon_{i} d_{i}^{\dagger} d_{i}+U \sum_{i} n_{i \sigma} n_{i, \bar{\sigma}}+\sum_{i<j, \sigma}\left(t_{i j} d_{i \sigma}^{\dagger} d_{j \sigma}+\right.$ h.c. $)$,

where $n_{i \sigma}=d_{i \sigma}^{\dagger} d_{i \sigma}$ is the occupation of $\operatorname{dot} i \in\{A, B, C\}$ with spin $\sigma$. Only one energy level $\epsilon_{i}=\epsilon$ is taken into account in each quantum dot - other levels are assumed to be much higher in energy. Parameter $U$ describes the Coulomb interaction between two electrons in the same orbital. We use compact notation for the hopping integrals: $t_{1}=t_{A C}, t_{2}=t_{A B}$, and $t_{3}=t_{B C}$, Figure 1 . The $\operatorname{dot} C$ is coupled to the left $(L)$ and the right $(R)$ electrode,

$$
H_{t}=\sum_{\alpha, k, \sigma}\left(V_{\alpha} c_{\alpha k \sigma}^{\dagger} d_{C \sigma}+\text { h.c. }\right)
$$

and $V_{\alpha}$ is the tunnel matrix element between lead $\alpha$ and $\operatorname{dot} C$. Electrons in the leads are described by a noninteracting Hamiltonian

$$
H_{\text {lead }}=\sum_{\alpha, k, \sigma}\left(\epsilon_{k} c_{\alpha k \sigma}^{\dagger} c_{\alpha k \sigma}+\text { h.c. }\right),
$$

where $\epsilon_{k}$ with wavevector $k$ is single-electron energy. $d_{i \sigma}^{\dagger}\left(d_{i \sigma}\right)$ and $c_{\alpha k \sigma}^{\dagger}\left(c_{\alpha k \sigma}\right)$ are the creation (annihilation) operators in the dot and in the lead, respectively. The leads have constant density of states $\rho=1 /(2 D)$, where $D$ is the half-bandwidth of the conduction band. The hybridization strength is $\Gamma=\pi \rho\left(\left|V_{L}\right|^{2}+\left|V_{R}\right|^{2}\right)$. Throughout this paper we assume $V_{L}=V_{R}$.

To properly describe the Kondo effect [42-45], impurity problems need to be solved using nonperturbative methods which correctly describe the screening of the impurity spin by the conduction-band electrons at low temperature scales. One such method is the numerical renormalization-group (NRG) [42,43,46-48]. It consists of a logarithmic discretization of the continuum of states of the conduction-band electrons, followed by a mapping to a one-dimensional chain Hamiltonian with exponentially decreasing hopping constants. As a consequence of these transformations, the hopping along such Wilson chain decreases exponentially, $t_{n} \approx \Lambda^{-n / 2}$, where $\Lambda>1$ is the discretization parameter and $n$ is the index of the site in the chain. This provides the opportunity to diagonalize the chain Hamiltonian iteratively and to keep only the states with the lowest lying energy eigenvalues, since the energy scales are separated (matrix elements between the states on vastly different energy scales are very small, thus may be neglected). Knowing the energy eigenstates and eigenvalues, we can calculate thermodynamical and dynamical quantities directly (e.g., spectral functions using their Lehmann representation). The method is reliable and rather accurate, easily generalizable to a range of different quantum impurity problems, including those with multiple impurities and several continuum channels.

The NRG calculations in this work were performed with a discretization parameter $\Lambda=2$, four values of the twist parameter $z$ to remove the discretization artefacts (oscillations in spectral functions) $[49,50]$ and the truncation energy cutoff of $E_{\text {cutoff }}=10 \omega_{N}$, where $\omega_{N}$ is the characteristic energy scale at the $N$ th NRG iteration; such cutoff is sufficient to guarantee the convergence of both thermodynamic and spectral properties. In this work we used the NRG Ljubljana code [44]. This package is an implementation of the NRG using Mathematica and $\mathrm{C}++$. The Mathematica part of the code is used for the initialization of the problem: using an input of Hamiltonian and operators of interest in the form of second-quantization expressions, it automatically generates the eigenvalues and eigenvectors in all symmetry-adapted subspaces of the full Fock space, as well as the matrix representations of all required operators. These are then used as the input to the numerically intensive part of the calculation programmed in $\mathrm{C}++$, which performs the iterative diagonalization and the transformations of operators, as well as the evaluation of the measurable quantities (expectation values, spectral 
weight distributions). The problem discussed in this work is a three-impurity single-channel quantum impurity problem which can be analyzed using relatively modest numerical requirements.

\section{Thermal entanglement}

The ability to entangle and to keep the entanglement of qubit pairs is one of the main challenges in quantum information processing tasks such as, e.g., dense coding, quantum cryptography, qubit teleportation or quantum computation. In general, it is quite common to find states that are at least to some extent entangled. To induce perfect entanglement of two isolated qubits (in a pure state), there are several possible approaches. In practice, however, the main challenge is to keep the states entangled also in the presence of the environment, in particular due to the effects of elevated temperature, fluctuating magnetic fields and the exchange and fluctuation of charge. For a pair of qubits, realized in a double quantum dot (DQD) system, it has been shown that the entanglement can be very robust in the presence of charge fluctuations as long as the temperature is sufficiently below the effective superexchange interaction among the qubits [19-21,26,27]. On the other hand, in the presence of external leads the formation of a many-body Kondo state is found in very low temperatures as a crucial mechanism responsible for the suppression of the entanglement.

Recently it was shown that in isolated TQD the entanglement is generated in the subspace of doublet states with different pairwise spin correlations for the ground and excited states [51]. The mixing of the states with an opposite spin orientation always monotonically destroys entanglement. Moreover, rotating an external electric field can create maximally entangled qubit pairs together with a separate spin that survives in a relatively wide temperature range providing robust pairwise entanglement generation at elevated temperatures.

Our main goal is to analyze the stability of the entanglement of such a TQD system coupled to external charge reservoirs, i.e., conducting leads. While a similar study of DQDs has shown that at low temperatures and low magnetic field the Kondo effect acts as a source of entanglement destruction [19-21,26,27], here - on the contrary the Kondo effect can induce perfect entanglement. Since the qubits are represented by electron spin, it is crucial that particular quantum dot is precisely singly occupied and with minimal charge fluctuations. While in an analogous DQD systems such perfect single occupancy can easily be achieved, here due to the lack of the particle-hole symmetry, single occupancy is achieved only to a good approximation $\left\langle n_{i \sigma}\right\rangle \approx 1$, while the total occupancy of TQD is set exactly to $\sum_{i \sigma}\left\langle n_{i \sigma}\right\rangle=3$ by tuning the model parameters.

We here quantify the entanglement by the Wootters concurrence $[52,53]$, for a pure two-qubit state $|A B\rangle=$ $\alpha_{\uparrow \uparrow}|\uparrow \uparrow\rangle+\alpha_{\uparrow \downarrow}|\uparrow \downarrow\rangle+\alpha_{\downarrow \uparrow}|\downarrow \uparrow\rangle+\alpha_{\downarrow \downarrow}|\downarrow \downarrow\rangle$ customarily defined by $C_{A B}=2\left|\alpha_{\uparrow \uparrow} \alpha_{\downarrow \downarrow}-\alpha_{\uparrow \downarrow} \alpha_{\downarrow \uparrow}\right|$. Two qubits are fully entangled, $C_{A B}=1$, if they are in one of the Bell states, given by $(|\uparrow \downarrow\rangle \pm|\downarrow \uparrow\rangle) / \sqrt{2}$ or $(|\uparrow \uparrow\rangle \pm|\downarrow \downarrow\rangle) / \sqrt{2}$.

In TQD, considered here, the qubits are coupled to a fermionic bath, therefore the two-qubit system $\mathrm{AB}$ is in a mixed state and an appropriate generalization for concurrence is given in terms of the reduced density matrix by the Wootters formula [52,53]. Moreover, there are also significant charge fluctuations in our system, thus the state may be outside the simple manifold of spin degrees of freedom. In any real system such charge fluctuations, as well as the coupling to the environment, are unavoidable and an interesting question arises: how do the deviations from the ideal case - pure spin system decoupled from external leads - influence the entanglement?

The concurrence can still be meaningfully and consistently defined for systems of the type considered here. In presence of axial spin symmetry, i.e., conserved square of the total spin $z$-axis projection, the concurrence can be given in a closed form in terms of two-electron correlation functions [19-21,26,27],

$$
\begin{aligned}
C_{A B} & =\frac{\max \left(0, C_{\uparrow \downarrow}, C_{\|}\right)}{P_{\uparrow \downarrow}+P_{\|}}, \\
C_{\uparrow \downarrow} & =2\left|\left\langle S_{A}^{+} S_{B}^{-}\right\rangle\right|-2 \sqrt{\left\langle P_{A}^{\uparrow} P_{B}^{\uparrow}\right\rangle\left\langle P_{A}^{\downarrow} P_{B}^{\downarrow}\right\rangle}, \\
C_{\|} & =\left|\left\langle S_{A}^{+} S_{B}^{+}\right\rangle\right|-2 \sqrt{\left\langle P_{A}^{\uparrow} P_{B}^{\downarrow}\right\rangle\left\langle P_{A}^{\downarrow} P_{B}^{\uparrow}\right\rangle}, \\
P_{\uparrow \downarrow} & =\left\langle P_{A}^{\uparrow} P_{B}^{\downarrow}+P_{A}^{\downarrow} P_{B}^{\uparrow}\right\rangle, \\
P_{\|} & =\left\langle P_{A}^{\uparrow} P_{B}^{\uparrow}+P_{A}^{\downarrow} P_{B}^{\downarrow}\right\rangle,
\end{aligned}
$$

where $S_{i}^{+}=\left(S_{i}^{-}\right)^{\dagger}=c_{i \uparrow}^{\dagger} c_{i \downarrow}$ is the electron spin raising operator for $\operatorname{dot} i=A, B$ and $P_{i}^{\sigma}=n_{i \sigma}\left(1-n_{i \sigma}\right)$ is the projection operator onto the subspace where dot $i$ is singly occupied by one electron with the spin $\sigma$. $P_{\|}$ and $P_{\uparrow \downarrow}$ are probabilities for the spins to be aligned in the same (parallel) and opposite (antiparallel) directions, respectively. This formalism is also applicable for the concurrence between other dots, e.g. $C_{C A}$ (concurrence between the $\operatorname{dot} C$ with the $\operatorname{dot} A$ ) and $C_{C B}$ (concurrence between the $\operatorname{dot} C$ with the $\operatorname{dot} B$ ). The required expectation values (spin correlators) can easily be computed using the NRG results at both zero and finite temperatures.

\section{Results}

\subsection{Thermodynamic quantities and transport}

In order to better understand the nature of the creation of the entanglement studied in this paper we also calculate several thermodynamic properties as follows.

(1) The temperature-dependent impurity contribution to the impurity magnetic susceptibility $[42,43]$

$$
\chi_{\mathrm{imp}}(T)=\frac{\left(g \mu_{B}\right)^{2}}{k_{B} T}\left(\left\langle S_{z}^{2}\right\rangle-\left\langle S_{z}^{2}\right\rangle_{0}\right),
$$


where $S_{z}$ is the $z$ component of the total spin of the whole system, while $\langle\ldots\rangle$ means the thermodynamic expectation values. The first expectation value refers to the system with dots, while the second (with the subscript 0) refers to the system without dots; $\mu_{B}$ is the Bohr magneton, $g$ is the $g$-factor, and $k_{B}$ is the Boltzmann constant. It should be noted that the combination $k_{B} T \chi_{\mathrm{imp}}(T) /\left(g \mu_{B}\right)^{2}$ can be considered as the effective magnetic moment of the impurity cluster.

(2) The impurity contribution to the entropy $[42,43]$

$$
S_{\mathrm{imp}}(T)=\frac{(E-F)}{T}-\frac{(E-F)_{0}}{T},
$$

where $E=\langle H\rangle=\operatorname{Tr}\left[H \exp \left(-H / k_{B} T\right)\right]$ and $F=$ $-k_{B} T \ln \operatorname{Tr}\left[\exp \left(-H / k_{B} T\right)\right]$. This quantity describes the effective number of degrees of freedom of the impurity cluster.

(3) Thermodynamic expectation values of various operators such as the on-site occupancy $\left\langle n_{i}\right\rangle$, local charge fluctuations $\left\langle\left(\delta n_{i}\right)^{2}\right\rangle=\left\langle n_{i}^{2}\right\rangle-\left\langle n_{i}\right\rangle^{2}$, and spin-spin correlations $\left\langle\mathbf{S}_{i} \cdot \mathbf{S}_{j}\right\rangle$.

(4) The electronic transport between the leads through $\operatorname{dot} C$, making use of the Meir-Wingreen formula [54]

$$
G(T)=G_{0} \pi \Gamma \int_{-\infty}^{+\infty} d \omega\left(-\frac{\partial f}{\partial \omega}\right) A_{C}(\omega, T)
$$

where $G_{0}=2 e^{2} / h$ is the conductance quantum, $f$ is the Fermi function, and $A_{C}(\omega, T)$ is the spectral function on the impurity $C$.

\subsection{Expected regimes}

Before presenting and discussing numerical results, it is worth recalling expected regimes. The behavior of the considered TQD system at various temperatures and interimpurity couplings is governed by the proximity to one of the following regimes: (i) three independent local moments; (ii) frustrated antiferromagnetic Heisenberg 3-sites ring, as the simplest representative of clusters exhibiting frustration and with significant generic features of many complex molecular systems in which various interactions participate; (iii) antiferromagnetic spin chain with total spin $S=1 / 2$; (iv) inter-impurity singlet formation between $\operatorname{dots} A$ and $B$ (with a decoupled spin-1/2 local moment at $\operatorname{dot} C) ;(\mathrm{v})$ inter-impurity triplet formation between $\operatorname{dots} A$ and $B$; (vi) single-impurity Kondo-effect at $\operatorname{dot} C$ (with two decoupled spin-1/2 local moments); (vii) two-stage Kondo-effect, with the first-stage screening process of $\operatorname{dot} C$ and the second-stage screening of $S=1$ between $\operatorname{dots} A$ and $B$ as a result of the reducing the net spin from $S=1$ to $S=1 / 2$; (viii) two-stage Kondo effect (TQD has the local spin $S=3 / 2$ ): the firststage screening of $S=3 / 2$ and reducing the net spin from $S=3 / 2$ to $S=1$, and the second-stage screening of $S=1$ to $S=1 / 2$.
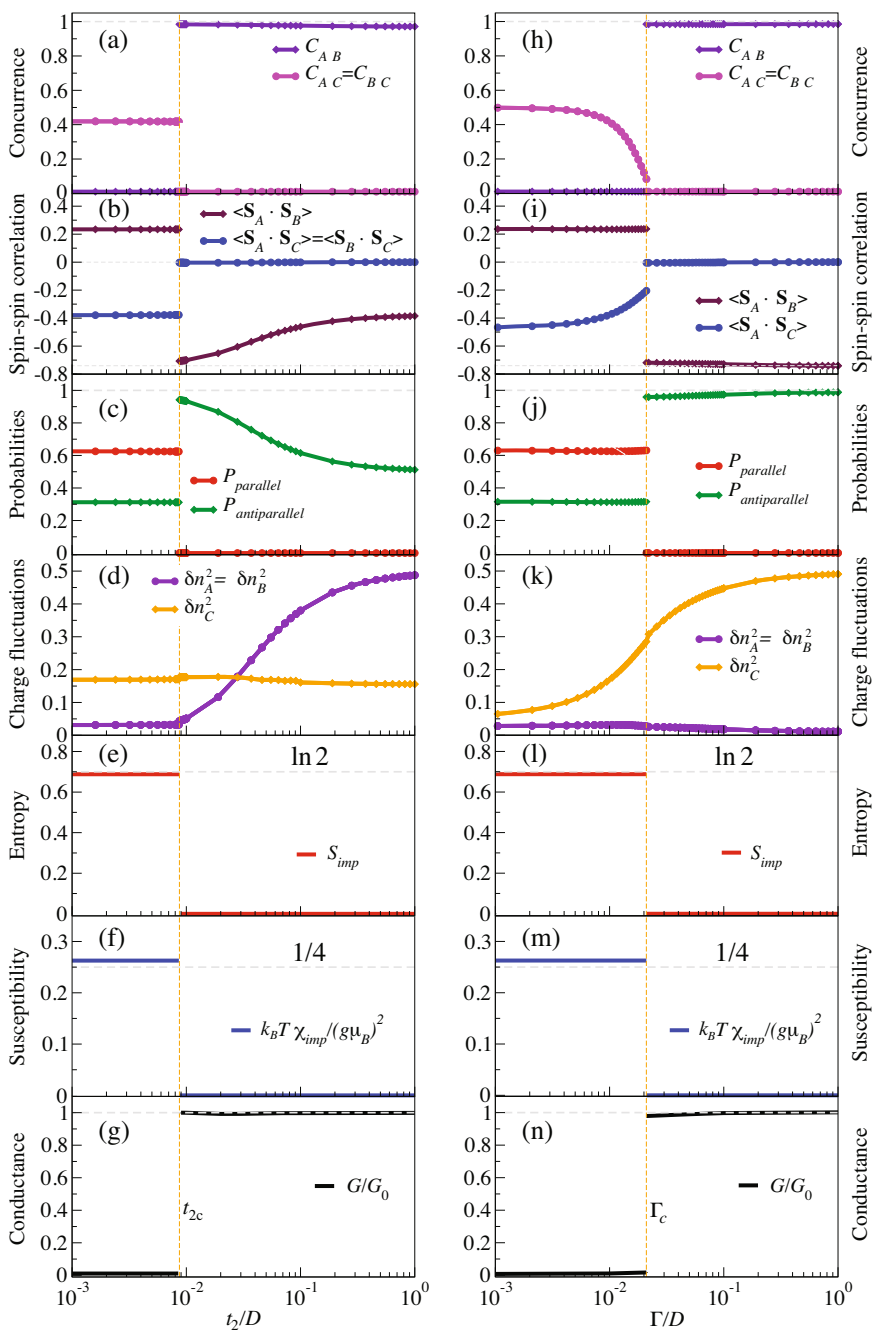

Fig. 2. Left panel: concurrence, (a), spin-spin correlations, (b), probabilities for parallel and antiparallel spin configuration, (c), charge fluctuations, (d), entropy $S_{\mathrm{imp}} / k_{B}$, (e), susceptibility $k_{B} T \chi_{\mathrm{imp}}(T) /\left(g \mu_{B}\right)^{2}$, (f), and conductance at temperature $T=10^{-6} D,(\mathrm{~g})$, as a function of $t_{2} / D$ for $U / D=0.1$, $\Gamma / D=0.01, \epsilon=-U / 2, t_{1,3} / D=0.01$. Right panel $(\mathrm{h})-(\mathrm{n})$ : results as in the left panel, but as a function of $\Gamma / D$ for $t_{2} / t_{1}=0.5$.

\subsection{Numerical analysis}

The entanglement properties of a dimer incorporated in an interacting system primarily depend on the spin properties of the side-coupled $\operatorname{dots} A$ and $B$. Our numerical analysis of the spin-spin correlation functions demonstrates a very robust singlet-triplet transition between the $\operatorname{dots} A$ and $B$, signaled by discontinuity of all system properties, see Figure 2. The two regimes are separated by a first order quantum phase transition (level crossing) as a function of $t_{2} / t_{1}$ which determines the ground state of the isolated TQD $[35,37,38,41]$. The same quantum phase transition has been previously found in a double quantum dot (DQD) system modeled as a pure spin system [55]. Entanglement properties of a qubit pair formed by spins on adjacent quantum dots are closely related to the spin-spin 
correlations $[26,27]$ therefore transitions between different spin configurations play a crucial role.

We first consider the strongly correlated regime with $U=10 \Gamma$ at temperature below the Kondo scale (essentially we are in the $T=0$ limit). Each of the dots is almost perfectly singly occupied. It should be noted that contrary to some DQD systems here exact average single occupancy of identical dots in general cannot be achieved for a common value of $\epsilon$ since the system is asymmetric and, furthermore, it is not particle-hole symmetric due to the lack of bipartiteness of the lattice. Expectation values $\langle\ldots\rangle$ in the concurrence formula equation (4) correspond to the thermal equilibrium of the system and consequently $\left\langle S_{A}^{+} S_{B}^{+}\right\rangle=0$. In vanishing magnetic field, the concurrence formula (4) simplifies further to

$$
C_{A B}=\max \left\{0,-2\left\langle\mathbf{S}_{A} \cdot \mathbf{S}_{B}\right\rangle /\left(P_{\uparrow \downarrow}+P_{||}\right)-1 / 2\right\} .
$$

Therefore, the concurrence is significant with increased spin-spin correlations in the range $-3 / 4 \leq\left\langle\mathbf{S}_{A} \cdot \mathbf{S}_{B}\right\rangle \leq-1 / 4$.

In Figure 2a we show the concurrence between the adjacent dots for a symmetric configuration with $t_{1}=t_{3}$. As expected, there are two regimes of interest, depending on the inter-dot couplings $\left(t_{2}\right.$ higher or lower compared to a transition value $t_{2 c}$ ), similar to the result of a recent analysis of thermal entanglement of isolated TQDs [51].

On one hand, for $t_{2}>t_{2 c}$ we get perfect entanglement between the dots $A$ and $B$, which are antiferromagnetically coupled, Figure $2 \mathrm{~b}$. Critical interdot coupling $t_{2 c}$ is equal to $t_{1}$ for decoupled quantum dots, $\Gamma=0$, and is slightly renormalized due to the coupling to the leads $[35,37,38,55]$. For increasingly large coupling $t_{2}$, the spin-spin correlations diminish due to charge fluctuations, Figure 2d, which reduce the probability for single occupation of the dots, Figure 2c. Interestingly, significant charge fluctuations $\delta n_{C}^{2}$ do not influence the sharp transition of the concurrence, which actually remains constant $C_{A B}=1$ up to very large values of $t_{2} / D$. This means that two electrons would form a perfectly entangled qubit pair if extracted one from the $\operatorname{dot} A$ and the other from $B$. The $\operatorname{dot} C$ is in the Kondo regime with electrons in the leads and it is effectively decoupled from the other two dots. Similar behavior, known as a dark-spin state, occurs in a TQD ring in the presence of an in-plane electric field due to decoupling of the spin in one of the dots [29-31].

On the other hand, for $t_{2}<t_{2 c}$, the concurrence is zero and ferromagnetic correlations between dots $A$ and $B$ dominate, forming an effective $S=1$ impurity at low temperatures which undergoes partial Kondo screening [41], yielding a residual uncompensated spin- $1 / 2$. This is a realization of the Kondo underscreening, well known from the single-channel spin-1 Kondo model.

In addition to the concurrence, spin and charge correlation functions, abrupt changes also occur in thermodynamic properties, which provides additional insight into the underlying physical picture. In particular, in Figure $2 \mathrm{e}$ we show the entropy change from $S_{\mathrm{imp}} / k_{B}=\ln 2$ to zero and the susceptibility from $\chi_{\mathrm{imp}}=1 / 4$ to 0 , Figure 2f. These changes are characteristic for a transition from a doublet ground state (of the full system) to a nondegenerate (singlet) ground state. Finally, at the transition the linear conductance changes from zero to $G=G_{0}$, Figure $2 \mathrm{~g}$. This is expected due to the decoupling of the dot $C$ from the entangled pair $A B$ - the conductance is unity as in the case of a single Anderson impurity.

The transition at $t_{2 c} \sim t_{1}$ is explained by the level crossing of the corresponding doublet eigenstates of the isolated TQD [29-31,35,37,38,51]. It should be noted that two-channel Kondo effects found in a quantum dot trimer coupled to the leads through two adjacent quantum dots [36-38] do not occur in the system considered here. However, for $t_{2}$ much lower than $t_{2 c}$ full-entanglement between the $\operatorname{dots} A$ and $B$ can be restored by a sufficient increase of the effective Kondo coupling of the $\operatorname{dot} C$ to the leads, leaving the dots $A$ and $B$ in the singlet state. In Figure 2h, we show how the concurrence $C_{A B}$ abruptly changes from zero to unity with increasing dot-lead hybridisation $\Gamma$. This is an alternative way of inducing the same quantum phase transition, as evident from the entropy, susceptibility and the conductance which all exhibit behavior analogous to the results presented as a function of $t_{2} / D$. Results presented in Figures $2 \mathrm{~h}-2 \mathrm{n}$ are thus analogous to those in Figures $2 \mathrm{a}-2 \mathrm{~g}$. The main distinction is the fact that dots $A$ and $B$ remain consistently decoupled from the rest of the system for the whole range of $\Gamma$, as is particularly clearly seen from the spin-spin correlations signaling triplet or singlet state for $\Gamma<\Gamma_{c}$ and $\Gamma>\Gamma_{c}$, respectively, while $\left\langle\mathbf{S}_{A} \cdot \mathbf{S}_{C}\right\rangle \sim 0$ due to the increase of $\delta n_{C}^{2}$ with increasing $\Gamma / D$. Even for extremely large values of fluctuations $\delta n_{C}^{2}$, the concurrence $C_{A B}$ remains constant above $\Gamma_{c}$. Charge fluctuations $\delta n_{A}^{2}$ remain low for the whole range of $\Gamma$, as expected.

\section{Discussion}

The results presented in Figure 2 are typical examples. In order to test the robustness of the results with respect to charge fluctuations on the dots, we performed a detailed numerical analysis summarized in the phase diagram in the parameter space $\left(U / \Gamma, t_{2} / t_{1}\right)$, see Figure 3 a. The moment of the $\operatorname{dot} C,\left\langle\mathbf{S}_{C}^{2}\right\rangle$, is represented as a contour plot (dashed lines). It is clear that for larger $\Gamma$ (i.e., smaller $U / \Gamma$ ) the moment is diminished which contributes to the renormalization of $t_{2 c}$.

The main reason for the renormalization can, in fact, be understood from an effective spin model by tracing out the empty and doubly occupied states, and representing the conduction band using a single spin $\mathbf{S}_{D}[29-31,35,37,38,51,55]$,

$$
H_{\text {eff }}=J_{1} \mathbf{S}_{A} \cdot \mathbf{S}_{C}+J_{2} \mathbf{S}_{A} \cdot \mathbf{S}_{B}+J_{3} \mathbf{S}_{B} \cdot \mathbf{S}_{C}+J_{D} \mathbf{S}_{C} \cdot \mathbf{S}_{D}
$$

with the exchange couplings $J_{1,2,3}=4 t_{1,2,3}^{2} / U$. First we consider a simple TQD system with $J_{D}=0$, i.e., without the coupling to spin $\mathbf{S}_{D}$. For the symmetric case with $J_{1}=J_{3}$ the eigenstates are two doublets and a quadruplet (which always lies the highest in energy). 

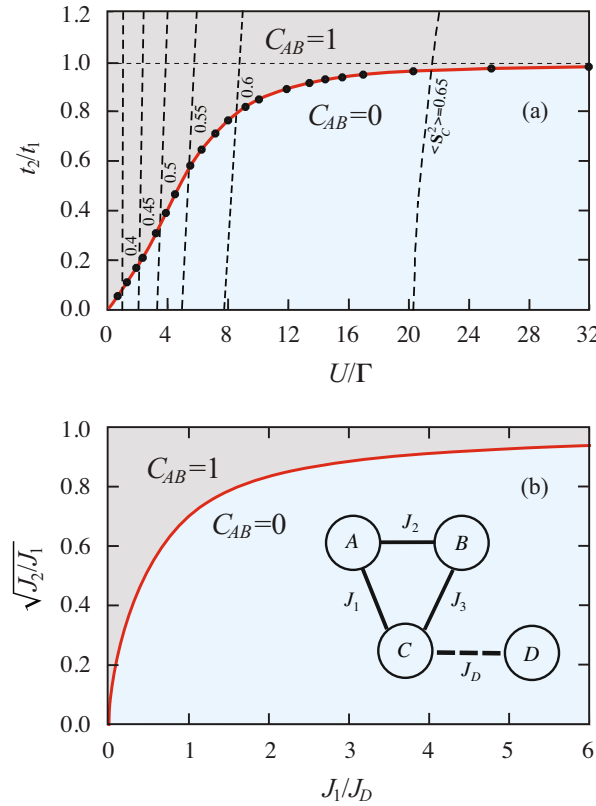

Fig. 3. (a) Phase diagram in the $\left(U / \Gamma, t_{2} / t_{1}\right)$ plane for $t_{1,3} / D=0.01$ and fixed $U / D=0.1$. Full red line (connecting calculated $t_{2 c} / t_{1}$, bullets) separates $C_{A B}=1$ (the Kondo phase) and $C_{A B}=0$ regions between two ground state configurations. Dashed lines represent constant values of local moment $\left\langle\mathbf{S}_{C}^{2}\right\rangle$. (b) Phase diagram in the $\left(J_{1} / J_{D}, \sqrt{J_{2} / J_{1}}\right)$ plane for an effective pure spin model, as an analogue of (a) with full line representing $\sqrt{J_{2 c} / J_{1}}$.

The ground states for the $z$-component of the total spin $S_{z}=1 / 2$ are given by:

$$
\begin{aligned}
\left|D_{1}\right\rangle & =\frac{\left(\left|\uparrow_{A} \downarrow_{B}\right\rangle-\left|\downarrow_{A} \uparrow_{B}\right\rangle\right) \otimes\left|\uparrow_{C}\right\rangle}{\sqrt{2}}, \\
\left|D_{2}\right\rangle & =\frac{2\left|\uparrow_{A} \uparrow_{B}\right\rangle \otimes\left|\downarrow_{C}\right\rangle-\left(\left|\uparrow_{A} \downarrow_{B}\right\rangle+\left|\downarrow_{A} \uparrow_{B}\right\rangle\right) \otimes\left|\uparrow_{C}\right\rangle}{\sqrt{6}} .
\end{aligned}
$$

The first state $\left|D_{1}\right\rangle$ is formed from the singlet state between the dots $A$ and $B$, for which the spin of the $\operatorname{dot} C$ is decoupled from the other dot spins:

$$
\left\langle D_{1}\left|\mathbf{S}_{C} \cdot \mathbf{S}_{A}\right| D_{1}\right\rangle=\left\langle D_{1}\left|\mathbf{S}_{C} \cdot \mathbf{S}_{B}\right| D_{1}\right\rangle=0 .
$$

The second state $\left|D_{2}\right\rangle$ is constructed from the triplet states between the dots $A$ and $B$ with $S_{z}=1$ and 0 , separately. The spin-spin correlation functions between the $\operatorname{dots} A$ and $B$ for the above two states are

$$
\left\langle D_{1}\left|\mathbf{S}_{A} \cdot \mathbf{S}_{B}\right| D_{1}\right\rangle=-3 / 4, \quad\left\langle D_{2}\left|\mathbf{S}_{A} \cdot \mathbf{S}_{B}\right| D_{2}\right\rangle=1 / 4 .
$$

Therefore, for the states $\left|D_{1}\right\rangle$ and $\left|D_{2}\right\rangle$ the spin-spin correlations between the $\operatorname{dots} A$ and $B$ are anti- and ferromagnetic, respectively. The eigenvalues are

$$
E_{1}=-12 t_{1}^{2} / U, \quad E_{2}=-4\left(4 t_{1}^{2}-t_{2}^{2}\right) / U .
$$

From the energy separation $E_{1}-E_{2}=J_{1}-J_{2}$ it is clear that $J_{2 c}=J_{1}$ is for large $U / \Gamma$ the critical value separating the regimes of the system in a quantum state where single electrons in $A$ and $B$ quantum dots are perfectly entangled - from the state, where these two electrons are completely unentangled, i.e., with $C_{A B}=0$.

Phase diagram presented in Figure $3 \mathrm{a}$ can be qualitatively understood also in the regime of lower $U / \Gamma$ where $t_{2 c}$ is significantly lower that $t_{1}$. A simple analysis of the effective Hamiltonian, equation (13), for $J_{D}>0$, i.e., for TQD coupled to an additional singly occupied quantum dot $D$, reveals a crossing of levels representing different ground states where the dots $A$ and $B$ form triplet or singlet configurations, if the interaction $J_{D}$ is below or above some $J_{D c}$, respectively. Critical value $J_{D c}$ can be given analytically,

$$
J_{D c}=\frac{\left(J_{1}-J_{2}\right)\left(J_{1}+2 J_{2}\right)}{2 J_{2}} .
$$

In Figure $3 \mathrm{~b}$ we show the phase diagram $\left(J_{1} / J_{D}, \sqrt{J_{2} / J_{1}}\right)$ where the full red line represents the separation between entangled and unentangled regimes. The topology of the dots is shown as an inset. Here $J_{D} / J_{1}$ plays the role of an effective Kondo coupling of TQD to the leads, $J_{D} \propto$ $\Gamma / U$. The $\operatorname{dots} A$ and $B$ for small $J_{2} / J_{1}$ but large $J_{D} / J_{1}$ thus exhibit perfect entanglement when dots $C$ and $D$ are forced to couple into a singlet, which can be considered as an example of the entanglement monogamy concept [56]. However, due to the substantial renormalization of the local moment $\left\langle\mathbf{S}_{C}^{2}\right\rangle$ in the limit of small values, $U / \Gamma<4$, the application of the pure spin model is not fully justified there. This is the reason for different behavior of critical $t_{2 c} / t_{1} \propto U / \Gamma$ and $\sqrt{J_{2 c} / J_{1}} \propto \sqrt{J_{1} / J_{D}}$ in this regime.

The quantum phase transition considered so far is of the first order. Here the entanglement, as measured by the concurrence, even in the presence of substantial charge fluctuations, is not significantly affected. Thus an interesting question arises, how the concurrence changes in the case of a broken symmetry configuration with $t_{1} \neq t_{3}$. Recently, the Kosterlitz-Thouless type of transition instead of the first order has been discussed for such a system [57].

We performed the analysis of symmetry breaking effects for the case where the hopping between quantum $\operatorname{dots} A C$ and $B C$ is modified to $t_{1,3}=t_{0} \pm \delta t$. We concentrated to the transition in the regime of parameters as in Figure 2, i.e., with $t_{0} / D=0.01$ with the corresponding $t_{2 c}$ slightly below $0.9 t_{0}$. The effects of asymmetry in a particular regime of parameters for a two impurity version of this model was studied also in reference [55]. The the main consequence of finite $\delta t$ is a smooth transition of the concurrence $C_{A B}$ from 0 to 1 for increasing $t_{2} / t_{0}$, Figure $4 \mathrm{a}$, with the corresponding spin-spin correlation $\left\langle\mathbf{S}_{A} \cdot \mathbf{S}_{B}\right\rangle$ also exhibiting a smooth transition, Figure $4 \mathrm{~b}$. Note also, that for $t_{2} \ll t_{0}$ the correlation is significantly reduced compared to the triplet value $1 / 4$.

These results can qualitatively be reproduced within the effective 4-QD model, inset to Figure 4b. As known from the analysis shown in Figure 3, for $\delta t=0$ the results of the effective model are identical to the full NRG result regarding the sharp transition of the correlations $\left\langle\mathbf{S}_{A} \cdot \mathbf{S}_{B}\right\rangle$. In the regime of broken symmetry with $J_{1,3}=J_{0} \pm \delta J$ the 


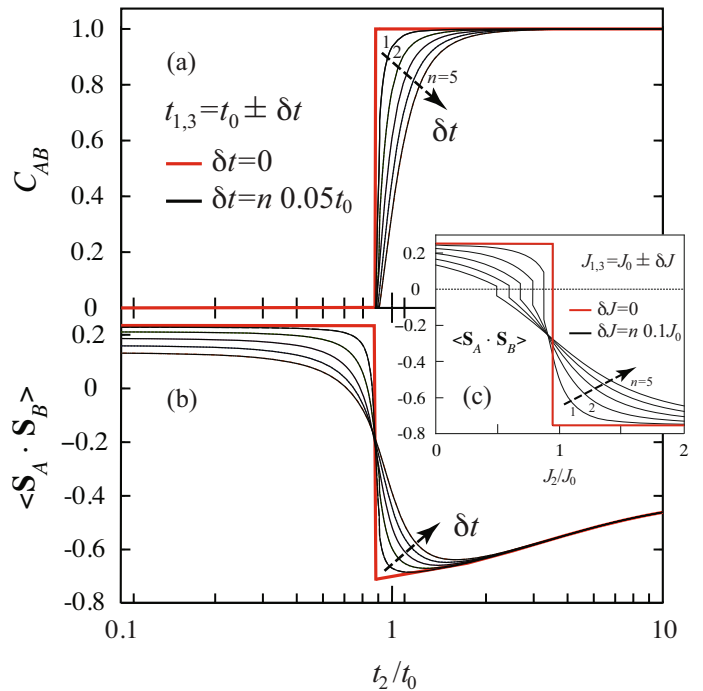

Fig. 4. (a) Concurrence $C_{A B}$ for the symmetry breaking case with $t_{0} / D=0.01$ for several values of $\delta t$, in increments $0.05 t_{0}$ (full black lines), compared to $\delta t=0$ (full red line) and with other parameters as in Figure 2. (b) Spin-spin correlations $\left\langle\mathbf{S}_{A} \cdot \mathbf{S}_{B}\right\rangle$ corresponding to $C_{A B}$ from Figure 3a. (c) Results for $\left\langle\mathbf{S}_{A} \cdot \mathbf{S}_{B}\right\rangle$ as obtained for the simplified model for $J_{0} / J_{D}=10$. Full black lines represent results for $\delta J$ ins increments $0.1 J_{0}$.

effective model adequately reproduces the correlations in the regime $J_{2} \rightarrow 0$. Note, however, the $J_{2}$-dependence of the correlations (and the concurrence, not shown here) calculated in the effective model does not change gradually, but it retains a sharp transition with the gap closing with increasingly larger $\delta J$, Figure 3c. The difference can mainly be attributed to the fact that in the effective model the change of the correlations is due to the change of the ground state while the full model exhibits a more complex many-body transition.

\section{Summary}

In summary, the spin entanglement of electron pairs in a triangular TQD, one dot being attached to non-interacting leads, is quantitatively analyzed in the regime of two competing many-body effects, namely the Kondo effect and the direct exchange interaction. In contrast to DQD configurations in which the Kondo effect suppresses the entanglement [19-21], in this case, the Kondo effect - in which a single spin in the $\operatorname{dot} C$ is screened by conduction electrons in an attached metallic lead - induces entanglement between the spins in the dots $A$ and $B$ and that such spin correlations can be signaled in charge transport mearusements. There are two regimes of interest, depending on the ratio of the interdot couplings $t_{2} / t_{1}$. On the one hand, for $t_{2}>t_{1}$, spins in the $\operatorname{dots} A$ and $B$ are always maximally entangled and the central $\operatorname{dot} C$ is in the Kondo regime with conduction leads, fully screened $S=1 / 2$ Kondo effect. On the other hand, for $t_{1}>t_{2}$, spins in the $\operatorname{dots} A$ and $B$ are in unentangled states due to the underscreened $S=1$ Kondo effect. Therefore, the entanglement is switched on due to sharp transition between the fully screened $S=1 / 2$ and underscreened $S=1$ Kondo effects. For stronger interaction regime $U / \Gamma \gg 1$ this result is expected as a consequence of the quantum phase transition observed in TQD with triangular topology $[35,37,38]$.

Our analysis reveals that the switching between unentangled and perfectly entangled qubit pairs $A$ and $B$ persists also in the regime of substantial charge fluctuations, either $\delta n_{A}^{2}$ or $\delta n_{C}^{2}$. In the regime of low $U / \Gamma$ there is a strong renormalization of $t_{2 c} \ll t_{1}$, which can be understood within a simple analytically solvable pure spin model where TDQ is anti-ferromagnetically coupled to an additional singly occupied quantum dot. The singlet formation between $C$ and $D$ acts as an entanglement switch between the $\operatorname{dots} A$ and $B$ in a similar manner as the formation of the Kondo singlet in the full TQD system that was analyzed numerically.

In the regime of broken symmetry between the quantum dots $A$ and $B$ there is no sharp transition between the entangled and unentangled states. Comparison with a simplified effective model of four magnetically coupled quantum dots reveals qualitative agreement in the sense that the transition is not sharp - it is only a smooth crossover. The quantum phase transition found in the symmetric configuration in this respect represents a singular point in the parameter space of the problem. Thus in a real quantum dot configuration a sharp transition would be expected only in the case of carefully tuned experimental conditions (gates).

The authors acknowledge A. Mitchell for the correspondence regarding the symmetry breaking effects. Financial support from the EU FP7 project: Marie Curie ITN NanoCTM, the Slovenian Research Agency under contract No. P1-0044, National Science Centre (Poland) under the contract DEC-2012/ 05/B/ST3/03208, and computing facilities at J. Stefan Institute are gratefully acknowledged.

\section{References}

1. R. Horodecki, P. Horodecki, M. Horodecki, K. Horodecki, Rev. Mod. Phys. 81, 865 (2009)

2. L. Amico, R. Fazio, A. Osterloh, V. Vedral, Rev. Mod. Phys. 80, 517 (2008)

3. V.N. Golovach, M. Borhani, D. Loss, Phys. Rev. B 74, 165319 (2006)

4. M.A. Nielsen, I.A. Chuang, Quantum Information and Quantum Computation (Cambridge University Press, Cambridge, 2001)

5. D. Loss, D.P. DiVincenzo, Phys. Rev. A 57, 120 (1998)

6. G. Burkard, D. Loss, D.P. DiVincenzo, Phys. Rev. B 59, 2070 (1999)

7. G. Burkard, D. Loss, E.V. Sukhorukov, Phys. Rev. B 61, R16303 (2000)

8. R. Hanson, L.P. Kouwenhoven, J.R. Petta, S. Tarucha, L.M.K. Vandersypen, Rev. Mod. Phys. 79, 1217 (2007)

9. E.A. Laird, J.M. Taylor, D.P. DiVincenzo, C.M. Marcus, M.P. Hanson, A.C. Gossard, Phys. Rev. B 82, 075403 (2010) 
10. L. Gaudreau, G. Granger, A. Kam, G.C. Aers, S.A. Studenikin, P. Zawadzki, M. Pioro-Ladriere, Z.R. Wasilewski, A. Sachrajda, Nat. Phys. 8, 54 (2012)

11. M. Busl, G. Granger, L. Gaudreau, R. Sánchez, A. Kam, M. Pioro-Ladriere, S.A. Studenikin, P. Zawadzki, Z.R. Wasilewski, A.S. Sachrajda, G. Platero, Nat. Nanotechnol. 8, 261 (2013)

12. E. Eriksson, H. Johannesson, Phys. Rev. B 84, 041107(R) (2011)

13. A. Rycerz, Eur. Phys. J. B 52, 291 (2006)

14. A. Montakhab, A. Asadian, Phys. Rev. A 77, 062322 (2008)

15. S.Y. Cho, R.H. McKenzie, Phys. Rev. A 73, 012109 (2006)

16. K. Le-Hur, P. Doucet-Beaupre, W. Hofstetter, Phys. Rev. Lett. 99, 126801 (2007)

17. J.H. Jefferson et al., Europhys. Lett. 74, 764 (2006)

18. G. Giavaras et al., Phys. Rev. B 74, 195341 (2006)

19. A. Ramšak, J. Mravlje, R. Žitko, J. Bonča, Phys. Rev. B 74, 241305(R) (2006)

20. J. Mravlje, A. Ramšak, T. Rejec, Phys. Rev. B 73, 241305(R) (2006)

21. T. Rejec, A. Ramšak, J.H. Jefferson, J. Phys.: Condens. Matter 12, L233 (2000)

22. A. Bayat, S. Bose, P. Sodano, Phys. Rev. Lett. 105, 187204 (2010)

23. A. Bayat, S. Bose, P. Sodano, Phys. Rev. B 81, 064429 (2010)

24. A. Bayat, S. Bose, P. Sodano, Phys. Rev. B 81, 100412(R) (2010)

25. A. Bayat, S. Bose, P. Sodano, H. Johannesson, Phys. Rev. Lett. 109, 066403 (2012)

26. A. Ramšak, I. Sega, J.H. Jefferson, Phys. Rev. A 74, 010304(R) (2006)

27. A. Ramšak, J. Mravlje, T. Rejec, A. Lautar, Europhys. Lett. 86, 40003 (2009)

28. M. Busl, R. Sánchez, G. Platero, Phys. Rev. B 81, 121306(R) (2010)

29. J. Łuczak, B.R. Bułka, J. Phys.: Condens. Matter 24, 375303 (2012)

30. B.R. Bułka, T. Kostyrko, J. Łuczak, Phys. Rev. B 83, 035301 (2011)

31. T. Kostyrko, B.R. Bułka, Phys. Rev. B 84, 035123 (2011)

32. R. Žitko, J. Bonča, A. Ramšak, T. Rejec, Phys. Rev. B 73, 153307 (2006)
33. R. Žitko, J. Bonča, Phys. Rev. B 74, 045312 (2006)

34. F. Delgado, Y.P. Shim, M. Korkusinski, P. Hawrylak, Phys. Rev. B 76, 115332 (2007)

35. W. Wang, Phys. Rev. B 78, 235316 (2008)

36. R. Žitko, J. Bonča, Phys. Rev. B 77, 245112 (2008)

37. A.K. Mitchell, T.F. Jarrold, D.E. Logan, Phys. Rev. B 79, 085124 (2009)

38. A.K. Mitchell, D.E. Logan, Phys. Rev. B 81, 075126 (2010)

39. G. Chiappe, E.V. Anda, R.L. Costa, E. Louis, Phys. Rev. B 81, 041310(R) (2010)

40. R. Žitko, J. Bonča, Phys. Rev. Lett. 98, 047203 (2007)

41. P.P. Baruselli, R. Requist, M. Fabrizio, E. Tosatti, Phys. Rev. Lett. 111, 047201 (2013)

42. K.G. Wilson, Rev. Mod. Phys. 47, 773 (1975)

43. R. Bulla, T. Costi, T. Pruschke, Rev. Mod. Phys. 80, 395 (2008)

44. NRG Ljubljana, open-source numerical renormalization group code, http://nrgljubljana.ijs.si/

45. A.C. Hewson, The Kondo Problem to Heavy-Fermions (Cambridge University Press, Cambridge, 1993)

46. T.A. Costi, A. Hewson, V. Zlatič, J. Phys.: Condens. Matter 6, 2519 (1994)

47. R.B. Bulla, T.A. Costi, D. Vollhardt, Phys. Rev. B 64, 045103 (2001)

48. W. Hofstetter, Phys. Rev. Lett. 85, 1508 (2000)

49. R. Žitko, Th. Pruschke, Phys. Rev. B 79, 085106 (2009)

50. R. Žitko, Comput. Phys. Commun. 180, 1271 (2009)

51. M. Urbaniak, S.B. Tooski, A. Ramšak, B.R. Bułka, Eur. Phys. J. B 86, 505 (2013)

52. W.K. Wootters, Phys. Rev. Lett. 80, 2245 (1998)

53. W.K. Wootters, Phys. Rev. Lett. 78, 5022 (1997)

54. Y. Meir, N.S. Wingreen, Phys. Rev. Lett. 68, 2512 (1992)

55. M. Vojta, R. Bulla, W. Hofstetter, Phys. Rev. B 65, 140405(R) (2002)

56. B.M. Terhal, IBM J. Res. Dev. 48, 71 (2004)

57. A.K. Mitchell, T.F. Jarrold, M.R. Galpin, D.E. Logan, J. Phys. Chem. B 117, 12777 (2013)

Open Access This is an open access article distributed under the terms of the Creative Commons Attribution License (http://creativecommons.org/licenses/by/4.0), which permits unrestricted use, distribution, and reproduction in any medium, provided the original work is properly cited. 